\title{
Conscious sedation services provided in secondary care for restorative dentistry
}

Conscious sedation services provided in secondary care for restorative dentistry in the UK: a survey

C. L. Morgan and A. M. Skelly Br Dent J 2005; 198: 631-635

Objectives

To assess the views of consultants in restorative dentistry on sedation services in secondary care for restorative dentistry and their involvement in the provision of this.

Design

Postal questionnaire survey in the UK.

Setting

Consultants in restorative dentistry.

Results

There was an 80\% response rate from 179 consultants. Among consultants in restorative dentistry there was a perceived need for sedation services in restorative dentistry within NHS hospitals other than for teaching purposes. Anxiety and level of trauma of dental treatment affected whether consultants felt it appropriate for patients to have such treatment under sedation. One third (48) of consultants treated patients under conscious sedation, a significant number of these held substantive posts and had graduated more recently. Of those (41) who provided treatment under conscious sedation in an NHS setting, most $(38,93 \%)$ provided treatment under intravenous sedation of whom only eight (21\%) acted as operator/sedationist. Nearly all consultants (135, 94\%) felt that specialist registrars in restorative dentistry should undergo some form of training in sedation.

\section{Conclusions}

Although consultants in restorative dentistry recognise the need for training in and the provision of sedation in secondary care for restorative dentistry, only one third of respondents currently provide this service.

\section{IN BRIEF}

- Among consultants in restorative dentistry there is a perceived need for sedation services to be provided in secondary care for restorative dentistry for selected patients and/or dental procedures.

- Currently only 41 out of 144 consultants (28\%) in restorative dentistry provide treatment under sedation within the NHS - which leaves many primary care practitioners with limited opportunity to refer patients for this type of secondary care.

- Almost all consultants (94\%) in restorative dentistry feel that specialist registrars in restorative dentistry should undergo training in sedation. This training need must be met if consultants in restorative dentistry are to provide conscious sedation services in secondary care in the future.

\section{COMMENT}

Since changes to the regulations governing the provision of general anaesthesia in 1998 and 2001 there has been a growing interest in the use of conscious sedation techniques in dentistry. At the same time there has been a focus on the standards of care for patients undergoing medical and dental procedures under sedation by clinicians who are not specialist anaesthetists. Dentistry has been pro-active through groups such as the Dental Sedation Teachers Group (DSTG), the Society for the Advancement of Anaesthesia in Dentistry (SAAD) and others in developing national guidelines for training and in trying to improve the standards relating to conscious sedation techniques.

In this interesting study based on responses to a postal questionnaire sent to consultants in restorative dentistry in the UK, an acceptable response rate of $80 \%$ was achieved. Opinions were sought on the type of patient and restorative treatment felt to be appropriate in the secondary care setting under sedation. In view of the large number of factors to be considered it is perhaps unsurprising that there was a wide range of opinions and some difficulty in answering this part of the questionnaire. Information on the number of consultants providing treatment under sedation was collected and although only a third provided this service the majority felt it was appropriate that specialist restorative services under sedation should be available to suitable patients. There were a greater number of younger consultants and those with NHS appointments who offered treatment under sedation, however the views of most consultants supported training in sedation for specialist registrars in restorative dentistry. There were differences of opinion in how extensive the training should be, however with the introduction of sedation training as a requirement by the Specialist Advisory Committee (SAC) in restorative dentistry in 2002 , it is to be hoped that all future consultants will recognise the advantages of standard conscious sedation techniques. The more specialist registrars that can reach a standard of competence in such techniques the better we are likely to be able to meet the needs of patients locally and able to pass on the necessary knowledge and skills to future trainees. In the discussion section the authors emphasise that sedation is not the only method for managing anxious dental patients and due regard should be given to all aspects of anxiety management. This perhaps should be kept in mind when designing this aspect of training programmes.

\section{A. F. Speirs, Consultant in Restorative Dentistry, Leeds Dental Institute}

doi:10.1038/sj.bdj.4812355 